\title{
轴向卤离子配位调控金属冠梄铽(III)配合物的慢磁弛豫行为
}

\author{
万瑞辰 ${ }^{\dagger}$ 伍思国 ${ }^{\dagger}$ 刘俊良 贾建华* \\ 黄国璋李泉文童明良* \\ (中山大学化学学院 生物无机与合成化学教育部重点实验室 广州 510275)
}

\begin{abstract}
摘要 以金属冠醚 $\left[15-\mathrm{MC}_{\mathrm{Ni}(\mathrm{II})}-5\right]$ 和卤素离子分别作为 $\mathrm{Tb}(\mathrm{III})$ 在赤道平面和轴向上的配体, 成功地组装了三例混合过渡 金属-稀土配合物 $\left\{\mathrm{TbNi}_{5} \mathrm{X}_{2}\right\}(\mathrm{X}=\mathrm{F}, \mathrm{Cl}, \mathrm{Br})$ ，其中 $\mathrm{TbNi}_{5} \mathrm{~F}_{2}$ 为首例双氟端基配位的稀土配合物. $\mathrm{X}$ 射线单晶衍射分析结果 表明, 配合物中 $\mathrm{Tb}$ (III) 离子采用具有高轴次对称性的五角双锥 $\left(D_{5 h}\right)$ 配位方式, 表明利用金属冠梄策略可以定向构筑含 五角双雉稀土基元的混合过渡金属-稀土配合物. 交流磁化率的测试结果表明, $\mathrm{Tb}$ (III)轴向的电荷分布对配合物的慢磁 弛豫行为有着重要的影响. 依次削弱轴向配体 $\mathrm{X}^{-}$的电负性, 在 $1 \mathrm{kOe}$ 外场下 $\left\{\mathrm{TbNi}_{5} \mathrm{~F}_{2}\right\}$ 和 $\left\{\mathrm{TbNi}_{5} \mathrm{Cl}_{2}\right\}$ 分别表现出场诱导 的单分子磁体和弱的慢磁弛豫行为, 而 $\left\{\mathrm{TbNi}_{5} \mathrm{Br}_{2}\right\}$ 则由于快速的磁量子隧穿而不表现出慢的磁弛豫行为.
\end{abstract}

关键词 稀土; 琙离子; 金属冠醚; 慢磁弛豫; 单分子磁体

\section{Modulation of Slow Magnetic Relaxation for Tb(III)-Metallacrown Complexes by Controlling Axial Halide Coordination}

\author{
Wan, Rui-Chen $^{\dagger} \quad$ Wu, Si-Guo ${ }^{\dagger} \quad$ Liu, Jun-Liang Jia, Jian-Hua* \\ Huang, Guo-Zhang Li, Quan-Wen Tong, Ming-Liang* \\ (Key Laboratory of Bioinorganic and Synthetic Chemistry of Ministry of Education, School of Chemistry, \\ Sun Yat-Sen University, Guangzhou 510275)
}

\begin{abstract}
Single-molecule magnets (SMMs), exhibiting magnetic bistability and slow magnetization relaxation, have fascinated scientific community for their promising applications in data storage and information processing. Great development has been achieved in lanthanide-based SMMs due to the unquenched orbital momentum and strong anisotropy of lanthanide ions. According to the crystal-field theory, the magnetic anisotropy of lanthanide ions arises from crystal-field splitting. Appropriate arrangement of coordination environment of lanthanide ion (including the local symmetry as well as the charge distribution) is key to design high-performance SMMs. However, it still remains a huge challenge to generate lanthanide-containing compounds with certain coordination environment. Taking advantage of metallacrown (MC) approach, herein a series of $3 \mathrm{~d}-4 \mathrm{f}$ complexes $\left\{\mathrm{TbNi}_{5} \mathrm{X}_{2}\right\}(\mathrm{X}=\mathrm{F}, \mathrm{Cl}, \mathrm{Br})$ were successfully isolated via solvothermal reactions. To obtain these complexes, a mixture of stoichiometric metal salt and quinaldichdroxamic acid with excess of pyridine derivative was dissolved in methanol and then heated at $75{ }^{\circ} \mathrm{C}$ for $2 \mathrm{~d}$. X-ray single-crystal diffraction analysis indicated that the $\mathrm{Tb}(\mathrm{III})$ site equatorially coordinates with [15- $\left.\mathrm{MC}_{\mathrm{Ni}(I I)}-5\right]$, whilst is axially capped by halide ions. As a result, the lanthanide ion possesses high axiality with a pentagonal bipyramid geometry $\left(D_{5 h}\right)$. Alternative current magnetic susceptibility data revealed that the electrostatic interactions between f-electrons and ligand electrons play an important role in modulating the magnetic relaxation dynamics. Maximizing the axial charge density in $\left\{\mathrm{TbNi}_{5} \mathrm{~F}_{2}\right\}$ where the $[\mathrm{F}-\mathrm{Ln}-\mathrm{F}]^{+}$moiety is firstly reported in lanthanide chemistry, the oblate $\mathrm{Tb}(\mathrm{III})$ is placed in a judicious crystal field. The out-of-phase signal of $\left\{\mathrm{TbNi}_{5} \mathrm{~F}_{2}\right\}$ shows obvious temperature and frequency dependence under 1 kOe applied dc field. Additionally, the slow magnetization relaxation of $\left\{\mathrm{TbNi}_{5} \mathrm{~F}_{2}\right\}$ can be fitted by the power law or Arrhenius plot with reversal barrier of $19.0 \mathrm{~K}$. By lowering the electrostatic interactions of axial ligation, the out-of-phase signal significantly weakens in $\left\{\mathrm{TbNi}_{5} \mathrm{Cl}_{2}\right\}$ and even vanishes in $\left\{\mathrm{TbNi}_{5} \mathrm{Br}_{2}\right\}$. The decline of magnetic anisotropy in $\left\{\mathrm{TbNi}_{5} \mathrm{Cl}_{2}\right\}$ and $\left\{\mathrm{TbNi}_{5} \mathrm{Br}_{2}\right\}$ accelerates the fast quantum tunneling of magnetization. The results demonstrate for the first time that the Off/Part/On slow magnetization relaxation can be modulated via the improvement of electronegativity of axial ligands.
\end{abstract}

Keywords lanthanide; $\mathrm{Tb}(\mathrm{III})$ ion; metallacrown; slow magnetic relaxation; single-molecule magnets

\footnotetext{
*E-mail: jiajh3@mail.sysu.edu.cn, tongml@mail.sysu.edu.cn

$\uparrow$ These authors contributed equally to this work.

Received March 19, 2020; published April 20, 2020.

Supporting information for this article is available free of charge via the Internet at http://sioc-journal.cn.

Project supported by the National Natural Science Foundation of China (Nos. 21771198, 21620102002), the Natural Science Foundation of Guangdong Province (No. 2017A030313059), the Science and Technology Plan of Guangzhou (No. 201806010192) and the Fundamental Research Funds for Central Universities (No. 19lgyjs31)

项目受国家自然科学基金(Nos. 21771198, 21620102002)、广东省自然科学基金(No. 2017A030313059)、广州市科技计划项目珠江科技新星专题(No. 201806010192)和高校基本科研业务费(No. 191gyjs31)资助.
} 


\section{1 引言}

二十多年前, Sessoli 等 ${ }^{[1,2]}$ 发现, 单分子磁体在 “阻 塞温度” 下表现出磁滞回线和慢磁弛豫行为, 分子被磁 化后其磁矩处于 “自旋向上” 或 “自旋向下” 状态，需 要翻越一个势能壁垒(即翻转能垒 $U_{\text {eff }}$ )才能恢复到热平 衡状态. 单分子磁体的磁双稳态类似于信息存储位元的 $\mid 0>$ 和 $\mid 1>$, 在超高密度信息存储方面有潜在的应用前 景. 阻塞温度 $\left(T_{\mathrm{B}}\right)$ 一般认为是磁滞回线的最高开口温度, 或被定义为弛豫时间达到 $100 \mathrm{~s}$ 时的温度, 其数值越高 越有利于磁体所存储信息的稳定性以及在该工作温度 下的可操作性 ${ }^{[3 \sim 6]}$. 与传统的块材磁体不同, 单分子磁 体表现出超顺磁性, 其慢磁弛豫行为经由自旋一晶格弛 豫机制和磁量子隧穿效应 $(\mathrm{QTM})$ 实现, 阻塞温度和翻转 能垒取决于分子的磁各向异性.

磁各向异性是设计高性能单分子磁体的关键要素, 增强磁各向异性、减小量子隧穿有助于提升磁双稳态的 工作温区. 稀土离子具有较大的磁矩和磁各向异性, 使 稀土单分子磁体成为近年来分子磁性领域的研究热 点 ${ }^{[7 \sim 15]}$. 但一方面由于具有较大的离子半径, 稀土离子 普遍青崃 8 9 配位, 其配体场的电荷分布大多近似球 形, 为稀土离子磁各向异性的调控造成困难 ${ }^{[16]}$. 另一方 面, 稀土离子的磁各向异性主要源于 $4 \mathrm{f}$ 电子与配位原 子的静电作用所引起的晶体场裂分. 因此, 为实现稀土 离子磁各向异性的最大化, 需要尽可能地降低 $4 \mathrm{f}$ 电子 $m_{J}$ 基态与配体电荷的静电互斥作用. 2011 年 Long 等 ${ }^{[17]}$ 根据 “点电荷静电模型” 以及 Sievers 的 $4 \mathrm{f}$ 电子电荷密 度分布形状一一扁圆或扁长形, 提出了针对稀土单离子 磁体的电荷密度匹配策略. 对前者而言, 比如 $\mathrm{Tb}(\mathrm{III}) 、$

Dy(III)、Ho(III)离子, 当配体电荷集中分布在轴向位置 时有利于获得较大的磁各向异性. 2013 年, 我们 ${ }^{[18]}$ 提出 并实践了有效调控稀土单分子磁体的翻转能垒和阻塞 温度等性能的对称性策略, 报道了首例具有五角双雉配 位几何的 $\left\{\mathrm{DyZn}_{2}\right\}$ 单分子磁体. 在该化合物中, 轴向配 位的酚羟基氧为 Dy(III)提供了强的单轴磁各向异性. 另 外, Dy(III)采用压扁的五角双锥配位构型, 减弱了晶体 场中横向组分的贡献, 翻转能垒和阻塞温度分别达到 $305 \mathrm{~cm}^{-1}$ 和 $11 \mathrm{~K}$. 当配位的甲醇分子脱去后形成了赝 $O_{h}$ 对称性的 $\left\{\mathrm{DyZn}_{2}\right\}_{\text {Oh }}$, 此时由于较大的横向晶体场导致 严重的磁量子隧穿, 单分子磁体的性能大幅下降. 基于 对称性策略, 我们陆续获得了多例单分子磁体性能优 异、热稳定性高的稀土配合物 ${ }^{[19 ~ 22]}$, 如 [Dy(bbpen)X] (X 为配位的卤素离子 $)$ 和 $\left[\mathrm{Dy}\left(\mathrm{OP}^{\mathrm{R}}\right)_{2}\left(\mathrm{H}_{2} \mathrm{O}\right)_{5}\right]^{3+}\left(\mathrm{OP}^{\mathrm{R}}\right.$ 为氧化 膦配体 $)^{[23,24]}$. 它们的翻转能垒和阻塞温度分别突破 712 $\mathrm{cm}^{-1}$ 和 $20 \mathrm{~K}$. 这种策略对较为少见的 $\mathrm{Ho}(\mathrm{III})$ 单分子磁 体也奏效, 比如 $\left[\mathrm{Ho}\left(\mathrm{OP}^{\mathrm{R}}\right)_{2}\left(\mathrm{H}_{2} \mathrm{O}\right)_{5}\right]^{3+}$ 的翻转能垒高达 237

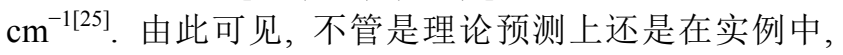
电荷分布的对称性控制是设计和合成高性能稀土单分 子磁体的有效手段.
众所周知, 以 $\mathrm{CH}_{2} \mathrm{CH}_{2} \mathrm{O}$ 为结构单元的常见冠醚分 子具有规整的空穴结构, 可以选择性识别半径合适的阳 离子并赋予其某种局部对称性. 但是, 此类配体缺乏刚 性，在与金属离子配位后容易发生不同程度的扭曲变 形，因此金属中心的配位几何通常难以保持理想的对称 性 ${ }^{[26,27]}$. 为解决这一难题, 我们想到了刚性更强的金属 冠醚化合物(MC), 巧妙地将金属冠醚特定的对称性 “精 准传递” 给中心的稀土离子, 成功获得了多例高稳定性 稀土单分子磁体, 比如 $\mathrm{Yb}[12-\mathrm{MC}-4]_{D 4 d^{[28]}}{ }^{[28}$ 以 $\mathrm{Ln}[15$ $\mathrm{MC}-6]_{D 6 h}(\mathrm{Ln}=\mathrm{Ce}, \mathrm{Nd})^{[29]}$. 由此看来, 金属冠醚不仅适 用于受关注最多的 Dy(III)-单分子磁体，还能被用来构 筑部分轻稀土 $\mathrm{Ce}(\mathrm{III})$ 或重稀土 $\mathrm{Yb}(\mathrm{III})$ 单分子磁体.

为了进一步拓展金属冠醚在设计合成稀土单分子 磁体中的应用, 本工作以 $4 \mathrm{f}$ 电子电荷密度呈扁圆形的 $\mathrm{Tb}(\mathrm{III})$ 与金属冠醚 $\left[15-\mathrm{MC}_{\mathrm{Ni}(\mathrm{II})}-5\right]$ 单元以及吡啶类辅助配 体组装得到了三例混合过渡金属-稀土配合物 $\left[\mathrm{TbNi}_{5} \mathrm{~F}_{2}-\right.$ (quinha) $)_{5}$ (3-chloropyridine) $\left.{ }_{10}\right]\left(\mathrm{ClO}_{4}\right)_{0.5} \mathrm{Cl}_{0.5} \bullet 10 \mathrm{H}_{2} \mathrm{O},\left[\mathrm{TbNi}_{5}\right.$ $\mathrm{Cl}_{2}$ (quinha) $)_{5}$ (pyridine $\left.)_{10}\right]\left(\mathrm{BPh}_{4}\right) \cdot 2 \mathrm{MeOH} \bullet$ pyridine $\cdot 5 \mathrm{H}_{2} \mathrm{O}$ 和 $\left[\mathrm{TbNi}_{5} \mathrm{Br}_{2} \text { (quinha) }\right)_{5}\left(3\right.$-bromopyridine) $\left.{ }_{10}\right] \mathrm{Br} \cdot 2 \mathrm{MeOH} \cdot \mathrm{H}_{2} \mathrm{O}$ $\left[\mathrm{H}_{2} \text { quinha }=\text { quinaldichydroxamic acid (喹啉羟肜酸) }\right]^{[28]}$. 三个配合物的内界组成与结构类似, 统一以 $\left\{\mathrm{TbNi}_{5} \mathrm{X}_{2}\right\}$ $(\mathrm{X}=\mathrm{F}, \mathrm{Cl}, \mathrm{Br})$ 表示. 在这些化合物中, $\mathrm{Tb}(\mathrm{III})$ 离子被封 装在金属冠醚环的中心而具有噟五次旋转轴. 本工作报 道了首例双氟端基配位的稀土配合物, 并尝试通过改变 轴向配位的卤素离子配体 $\mathrm{X}^{-}$以调节 $\mathrm{Tb}(\mathrm{III})$ 的单轴磁各 向异性，进而调控配合物的慢磁弛豫行为.

\section{2 结果与讨论}

\section{1 配体配位模式}

配合物中主要采用的有机配体为喹啉羟弜酸, 它与 金属的配位模式如图 1a 所示. 喹啉羟肜酸在碱性条件 去质子化后与 $\mathrm{Ni}(\mathrm{II})$ 离子形成金属冠醚 $\left[15-\mathrm{MC}_{\mathrm{Ni}}\right.$ (II)-5](图 $1 b$ ), 吡啶类辅助配体位于 $\mathrm{Ni}(\mathrm{II})$ 离子的上下方 (以 $\mathrm{TbNi}_{5} \mathrm{~F}_{2}$ 为例, 图 $\left.2 \mathrm{~b}\right) . \mathrm{Tb}(\mathrm{III})$ 离子位于金属冠醚赤道面 中心并与赤道面的五个氧原子配位, 两个卤素离子从上 下轴向的位置与 $\mathrm{Tb}(\mathrm{III})$ 离子配位(图 1c). (a)

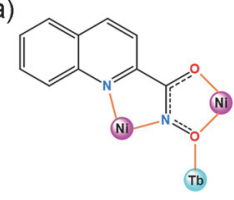

(b)

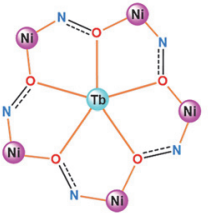

(c)

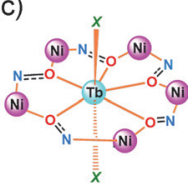

图 1 配体与金属离子的配位模式

Figure 1 Coordination mode of ligands with metal atoms

\section{2 晶体结构}

$X$ 射线单晶衍射分析结果表明，三个配合物 $\left\{\mathrm{TbNi}_{5} \mathrm{X}_{2}\right\}$ 均结晶于三斜晶系 $P-1$ 空间群(表 $\mathrm{S} 1$ ). 晶胞中 每个最小不对称单元包含一个 $\mathrm{Tb}(\mathrm{III})$ 离子，两个轴向配 位的 $\mathrm{X}^{-}$离子, 以及由五个 $\mathrm{Ni}(\mathrm{II})$ 离子、五个去质子化的 
喹啉羟肜酸和十个吡啶类分子形成的金属冠醚 $\left[15-\mathrm{MC}_{\mathrm{Ni}(\mathrm{II})}-5\right]$ ( 以 $\mathrm{TbNi}_{5} \mathrm{~F}_{2}$ 为例, 图 $2 \mathrm{a} \sim 2 \mathrm{c}$ ). 喹啉羟肟酸 上的两个 $\mathrm{N}$ 原子和两个 $\mathrm{O}$ 原子分别螯合一个 $\mathrm{Ni}(\mathrm{II})$ 原子, 形成 $\mathrm{Ni}-\mathrm{N}-\mathrm{O}$ 结构单元. 五个 $\mathrm{Ni}-\mathrm{N}-\mathrm{O}$ 首尾相连形 成近似平面的金属冠醚 $\left[15-\mathrm{MC}_{\mathrm{Ni}(\mathrm{II})}-5\right]$. $\mathrm{Tb}(\mathrm{III})$ 处于该金 属冠醚中心, 分别与五个肟基 $\mathrm{O}$ 原子和两个卤素离子在 赤道平面和轴向位置配位, 形成了五角双锥配位几何构 型. 金属冠醚平面两侧各有五个吡啶类辅助配体通过 $\mathrm{N}$ 原子与 $\mathrm{Ni}(\mathrm{II})$ 离子配位, 所有 $\mathrm{Ni}(\mathrm{II})$ 采用 $\left[\mathrm{N}_{4} \mathrm{O}_{2}\right]$ 的八面体 配位模式, 有利于增强金属冠醚的稳定性. 此外, 由吡 啶类配体构成的空腔还能限制 $\mathrm{X}^{-}$离子与 $\mathrm{Tb}(\mathrm{III})$ 离子的 线性配位角度，进而影响 $\mathrm{Tb}(\mathrm{III})$ 离子的配位环境. (a)

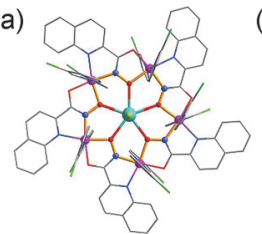

(d)

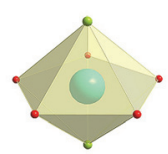

(b)

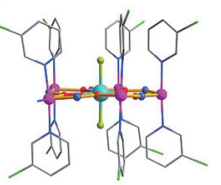

(e)

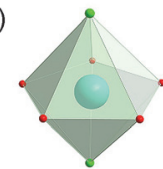

(c)
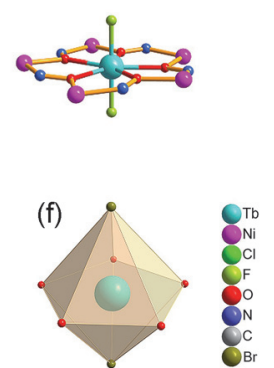

图 $2 \mathrm{TbNi}_{5} \mathrm{~F}_{2}$ 的晶体结构 $(\mathrm{a} \sim \mathrm{c})$ 和 $\mathrm{Tb}(\mathrm{III})$ 离子的配位多面体 $(\mathrm{d}$ : $\left.\mathrm{TbNi}_{5} \mathrm{~F}_{2} ; \mathrm{e}: \mathrm{TbNi}_{5} \mathrm{Cl}_{2} ; \mathrm{f:} \mathrm{TbNi}_{5} \mathrm{Br}_{2}\right)$

Figure 2 Crystal structure of $\mathrm{TbNi}_{5} \mathrm{~F}_{2}(\mathrm{a} \sim \mathrm{c})$ and coordinate polyhedron of $\mathrm{Tb}(\mathrm{III})$ ion (d: $\left.\mathrm{TbNi}_{5} \mathrm{~F}_{2} ; \mathrm{e}: \mathrm{TbNi}_{5} \mathrm{Cl}_{2} ; \mathrm{f}: \mathrm{TbNi}_{5} \mathrm{Br}_{2}\right)$

Part of ligands and all $\mathrm{H}$ atoms are omitted for clarity

表 $\mathrm{S} 2$ 列出了 $\left\{\mathrm{TbO}_{5} \mathrm{X}_{2}\right\}$ 多面体的部分键长键角数据. 赤道面的 $\mathrm{Tb}-\mathrm{O}$ 键长范围在 $0.2452(3) \sim 0.2472(3) \mathrm{nm}$ $\left(\mathrm{TbO}_{5} \mathrm{~F}_{2}\right) 、 0.2420(3) \sim 0.2443(3) \mathrm{nm} \quad\left(\mathrm{TbO}_{5} \mathrm{Cl}_{2}\right)$ 和 $0.2429(6) \sim 0.2461(6) \mathrm{nm}\left(\mathrm{TbO}_{5} \mathrm{Br}_{2}\right), \mathrm{O}-\mathrm{Tb}-\mathrm{O}$ 的键角 范围分别为 $71.76(10)^{\circ} \sim 72.59(11)^{\circ}\left(\mathrm{TbO}_{5} \mathrm{~F}_{2}\right)$ 、 $71.69(8)^{\circ} \sim 72.30(9)^{\circ}\left(\mathrm{TbO}_{5} \mathrm{Cl}_{2}\right)$ 和 $71.5(2)^{\circ} \sim 72.7(2)^{\circ}$ $\left(\mathrm{TbO}_{5} \mathrm{Br}_{2}\right)$. 轴向 $\mathrm{X}^{-}$离子与 $\mathrm{Tb}(\mathrm{III})$ 离子近似线形配位, $\mathrm{X}-\mathrm{Tb}-\mathrm{X}$ 的键角为 $178.91(12)^{\circ}\left(\mathrm{TbO}_{5} \mathrm{~F}_{2}\right) 、 176.66(3)^{\circ}$ $\left(\mathrm{TbO}_{5} \mathrm{Cl}_{2}\right)$ 和 $172.20(6)^{\circ}\left(\mathrm{TbO}_{5} \mathrm{Br}_{2}\right)$, 非常接近理想的线性 配位键角 $180^{\circ}$, 与具有类似结构的 Dy(III)-和 Ho(III)-单 分子磁体的轴向 $\mathrm{O}-\mathrm{Ln}-\mathrm{O}$ 角度 $\left(175.79^{\circ}, 179.04^{\circ}\right.$, $\left.177.9^{\circ}\right)$ 接近 $\left.{ }^{[23} 25\right]$. 从上述数据可以看出, 七配位的 $\left\{\mathrm{TbO}_{5} \mathrm{X}_{2}\right\}$ 多面体很接近五角双雉构型, 利用 Shape 软件 对其构型进行模拟(表 S3, CShM (the continuous shape measures)的数值越小表明 $\mathrm{Tb}(\mathrm{III})$ 离子的配位环境越接 近该几何构型). 计算结果表明, 三个配合物中 $\mathrm{Tb}(\mathrm{III})$ 离 子的配位几何构型均为赝 $D_{5 h}$ 对称性的五角双雉构型, 其中 $\mathrm{TbNi}_{5} \mathrm{Cl}_{2}$ 最接近 $D_{5 h}$ 对称性. 值得注意的是, 由于 $\mathrm{F}^{-}$离子的电负性高, $\mathrm{TbNi}_{5} \mathrm{~F}_{2}$ 的 $\mathrm{F}^{-}$离子在与 $\mathrm{Tb}(\mathrm{III})$ 离子 配位的同时, 还与晶格中的水分子作用, 形成相对稳定 的氢键 $(F \cdots O=0.2719 \mathrm{~nm}$, 图 $\mathrm{S} 4)$.
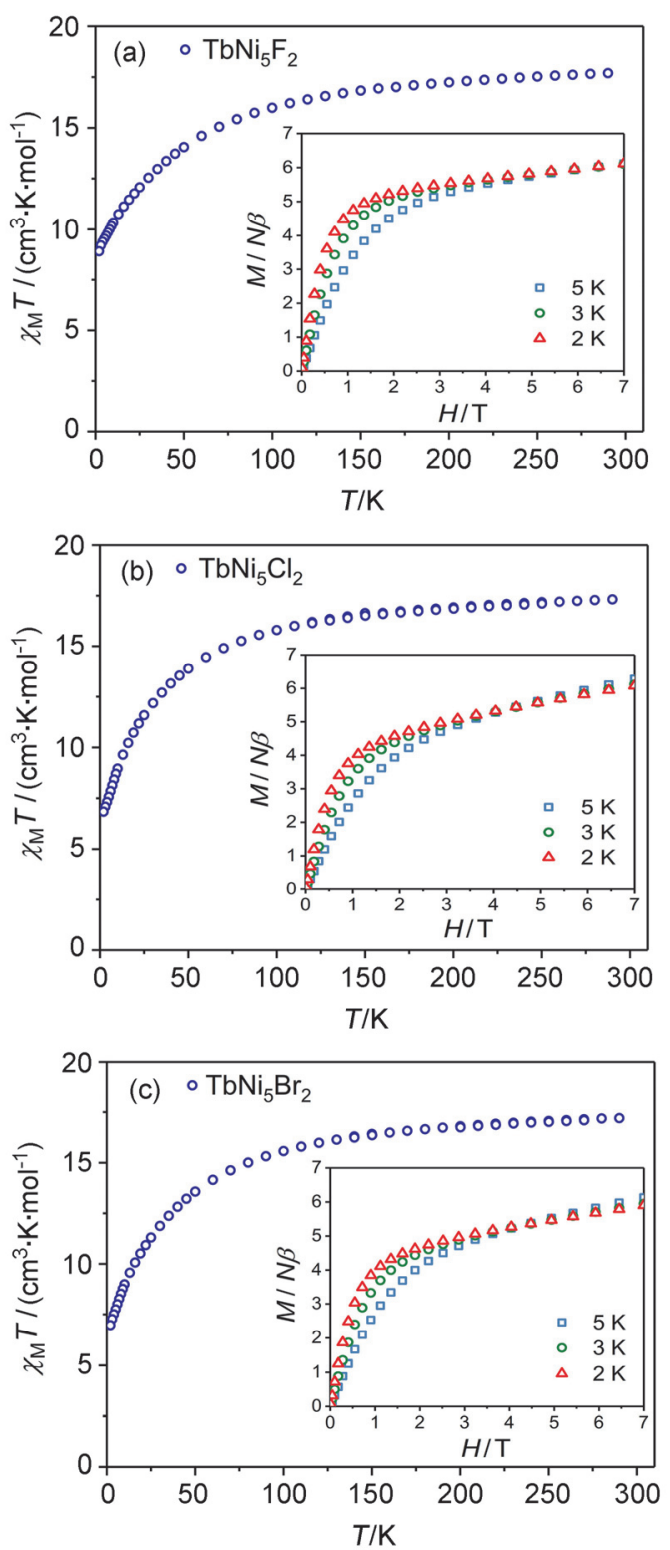

图 3 变温直流摩尔磁化率曲线和不同温度下变场磁化强度曲线 Figure 3 Temperature dependent direct-current (DC) molar magnetic susceptibilities $\left(\chi_{\mathrm{M}} T\right.$ vs. $\left.T\right)$

(a) $\mathrm{TbNi}_{5} \mathrm{~F}_{2}$, (b) $\mathrm{TbNi}_{5} \mathrm{Cl}_{2}$, (c) $\mathrm{TbNi}_{5} \mathrm{Br}_{2}$. Inset is the field dependent magnetization $(M$ vs. $H)$ at 2,3 and $5 \mathrm{~K}$

\section{3 磁学性质}

首先在 $1 \mathrm{kOe}$ 外加磁场下对配合物进行变温直流磁 化率测试(图 3). 室温下的 $\chi_{\mathrm{M}} T$ 值分别为 $17.69\left(\mathrm{TbNi}_{5} \mathrm{~F}_{2}\right)$, $17.32\left(\mathrm{TbNi}_{5} \mathrm{Cl}_{2}\right), 17.20\left(\mathrm{TbNi}_{5} \mathrm{Br}_{2}\right) \mathrm{cm}^{3} \cdot \mathrm{K}^{\prime} \cdot \mathrm{mol}^{-1}$, 接近一 个自由 $\mathrm{Tb}(\mathrm{III})$ 离子 $\left({ }^{7} F_{6}, g=3 / 2, \chi_{\mathrm{M}} T\right.$ 高温极限值为 11.82 $\mathrm{cm}^{3} \cdot \mathrm{K} \cdot \mathrm{mol}^{-1}$ ) 和五个纯自旋 $\mathrm{Ni}(\mathrm{II}$ ) 离子(对单个 $\mathrm{Ni}(\mathrm{II}$ )离子 来说, $S=1,\left\{\mathrm{Ni}_{5}\right\}$ 的 $\chi_{\mathrm{M}} T$ 高温极限值为 $\left.5 \mathrm{~cm}^{3} \cdot \mathrm{K} \cdot \mathrm{mol}^{-1}\right)$ 的 理论值之和 $16.82 \mathrm{~cm}^{3} \cdot \mathrm{K} \cdot \mathrm{mol}^{-1[19,30]}$. 随着温度的降低, $\chi_{\mathrm{M}} T$ 值缓慢降低, 当温度达到 $2 \mathrm{~K}$ 时, 它们的 $\chi_{\mathrm{M}} T$ 值分 别为 $9.86,6.84,6.96 \mathrm{~cm}^{3} \cdot \mathrm{K} \cdot \mathrm{mol}^{-1}$. 这与最近报道的类似 化合物 $\left\{\mathrm{EuCu}_{5}\right\}$ 中五个 $\mathrm{Cu}(\mathrm{II})$ 之间存在反铁磁相互作用 
相一致 ${ }^{[31]},\left\{\mathrm{TbNi}_{5} \mathrm{X}_{2}\right\}$ 的 $\mathrm{Ni}_{5}$ 环也存在强的 $\mathrm{Ni} \cdots \mathrm{Ni}$ 反铁磁 相互作用. 从以上分析得出, 造成 $\chi_{\mathrm{M}} T$ 值随温度降低而 减少的主要因素包括 $\left\{\mathrm{Ni}_{5}\right\}$ 环存在较强的反铁磁相互作 用和 Stark 亚能级激发态上分子的热布居减少. 图 3 内 插图为化合物在 2,3 和 $5 \mathrm{~K}$ 的等温磁化强度数据. 在 $0 \sim 7 \mathrm{~T}$ 外加磁场下, 磁化强度随磁场强度的增大而上 升. 当到达最大磁场 $7 \mathrm{~T}$ 时, 磁化强度分别上升到 6.12 $\left(\mathrm{TbNi}_{5} \mathrm{~F}_{2}\right), 6.28\left(\mathrm{TbNi}_{5} \mathrm{Cl}_{2}\right), 6.13\left(\mathrm{TbNi}_{5} \mathrm{Br}_{2}\right) \mathrm{N} \beta$, 从增长 趋势可见均未达到磁饱和状态, 表明配合物分子内存在 强的磁各向异性以及反铁磁相互作用.

在动态磁行为方面, 首先以 $\mathrm{TbNi}_{5} \mathrm{Cl}_{2}$ 为研究对象. 在无外加直流磁场下对其进行交流磁化率测试, 收集不 同频率下温度范围从 $2 \sim 8 \mathrm{~K}$ 的摩尔交流磁化率实部 $\left(\chi_{M}^{\prime}\right)$ 和虚部 $\left(\chi^{\prime \prime}{ }_{M}\right)$ 数据. $\chi^{\prime \prime}{ }_{M}$ 值随着温度的降低而逐渐增 大, 但即使频率提高近 10 倍时其数值也基本不变, 没有 表现出频率依赖性(图 S5). 而 $\chi^{\prime \prime}{ }_{\mathrm{M}}$ 值随着温度的降低并 没有响应, 就算频率升高到 $999 \mathrm{~Hz}$, 其数值也没有明显 的变化. 究其原因, 主要是存在严重的磁量子隧穿效应. 据我们所知, 隧穿速率与磁场的平方呈负相关, 一定强 度的直流磁场能有效地抑制磁量子隧穿行为. 因此, 将 外加直流磁场升高到 $1 \mathrm{kOe}$ 再次收集交流磁化率数据如 图 $4 \mathrm{a}$ 所示. 可以看出, 在温度低于 $4 \mathrm{~K}$ 时, $\chi^{\prime{ }_{\mathrm{M}}}$ 曲线数值 开始增大并伴随较为明显的频率依赖性, 表明在 $1 \mathrm{kOe}$ 直流场下 $\mathrm{TbNi}_{5} \mathrm{Cl}_{2}$ 具有慢磁弛豫行为.

从 $\mathrm{TbNi}_{5} \mathrm{Cl}_{2}$ 的晶体结构数据可以看出, 赤道面的 $\mathrm{Tb}-\mathrm{O}$ 键长均比轴向的 $\mathrm{Tb}-\mathrm{Cl}$ 键长短得多 (差值 $>0.02$ $\mathrm{nm})$. 根据 Long 等 ${ }^{[17]}$ 的推论, 这样的晶体场环境与 $\mathrm{Tb}(\mathrm{III})$ 离子扁圆形的电子电荷密度分布形状并不匹配. 本课题组近期在 $D_{5 h}$ 对称性控制方面的成果也表明, 轴 向键长越短越有利于抑制量子隧穿效应. 对于扁圆型的 $\mathrm{Tb}(\mathrm{III})$ 离子来说, 尽可能缩短轴向的 $\mathrm{Tb}-\mathrm{X}$ 键长, 使轴 向电荷分布向 $\mathrm{Tb}(\mathrm{III})$ 离子靠近. 因此, 保持原有金属冠 醚[15- $\mathrm{MC}_{\mathrm{Ni}(\mathrm{II})^{-}}$] 不变, 将轴向配位的 $\mathrm{Cl}^{-}$离子换成半径 更小的 $\mathrm{F}^{-}$离子, 得到了类似物 $\mathrm{TbNi}_{5} \mathrm{~F}_{2}$. 它的轴向 $\mathrm{Tb}-$ $\mathrm{F}$ 平均键长为 $0.2152(3) \mathrm{nm}$, 与 $\mathrm{TbNi}_{5} \mathrm{Cl}_{2}$ 的平均值 $0.2676(1) \mathrm{nm}$ 相比足足缩短了 $0.05 \mathrm{~nm}$ (表 S2).

$\mathrm{TbNi}_{5} \mathrm{~F}_{2}$ 的交流磁化率测试结果表明, 在无外加直 流磁场下交流磁化率虚部信号 $\chi^{\prime \prime}{ }_{\mathrm{M}}$ 曲线就已表现出频率 依赖性(图 4b). 但在所测温度范围内 $\mathrm{TbNi}_{5} \mathrm{~F}_{2}$ 的 $\chi^{\prime \prime}{ }_{\mathrm{M}}$ 曲线 没有出现峰值, 主要归因于此温度范围分子内存在较强 的量子隧穿效应. 同样对其交流磁化率的测试施加 1 $\mathrm{kOe}$ 的外加直流场, 所得到的变温磁化率曲线在 $5 \mathrm{~K}$ 左 右出现峰值, 并随着频率的降低向低温区移动(图 5a). 等温磁化率的 $\chi^{\prime \prime}{ }_{M}$ 曲线表明 $\mathrm{TbNi}_{5} \mathrm{~F}_{2}$ 具有频率依赖性, 温度为 $2 \mathrm{~K}$ 时 $\chi^{\prime \prime}{ }_{\mathrm{M}}$ 曲线在频率略低于 $10 \mathrm{~Hz}$ 的位置出现 峰值(图 5b). 随着温度的升高, 峰位逐渐向高频区移动, 当温度高于 $7 \mathrm{~K}$ 时, $\chi^{\prime{ }_{\mathrm{M}}}$ 峰值超出仪器的交流频率测试上 限 $(1000 \mathrm{~Hz}) . \mathrm{TbNi}_{5} \mathrm{~F}_{2}$ 的交流磁化率曲线具有明显的频
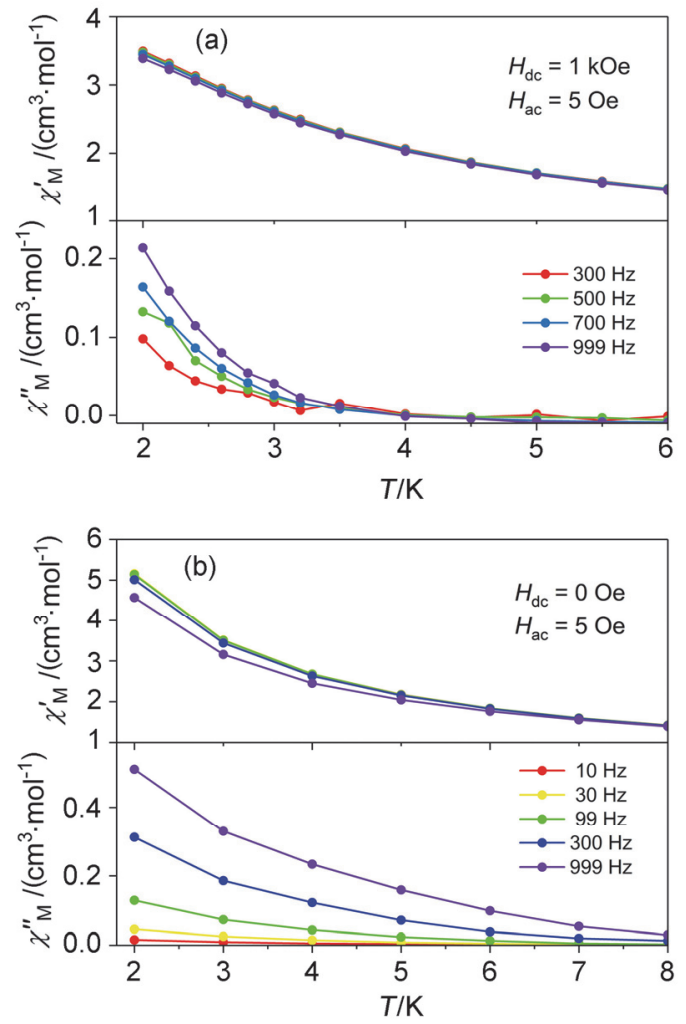

图 4 在 $1 \mathrm{kOe}$ 直流场下 $\mathrm{TbNi}_{5} \mathrm{Cl}_{2}$ (a) 和零外加场下 $\mathrm{TbNi}_{5} \mathrm{~F}_{2}(\mathrm{~b})$ 的变温 交流摩尔磁化率曲线

Figure 4 Temperature-dependent alternating-current (AC) molar magnetic susceptibilities of $\mathrm{TbNi}_{5} \mathrm{Cl}_{2}$ (a) under $1 \mathrm{kOe} \mathrm{DC}$ field and $\mathrm{TbNi}_{5} \mathrm{~F}_{2}$ (b) in zero DC field.

率依赖性且在 $2 \sim 7 \mathrm{~K}$ 温区出现峰值, 表明在 $1 \mathrm{kOe}$ 外场 下 $\mathrm{TbNi}_{5} \mathrm{~F}_{2}$ 是一例典型的单分子磁体.

将 $2 \sim 7 \mathrm{~K} 、 1 \mathrm{kOe}$ 直流场下 $\mathrm{TbNi}_{5} \mathrm{~F}_{2}$ 的交流磁化率 虚部信号 $\left(\chi^{\prime \prime}{ }_{M}\right)$ 对实部信号 $\left(\chi_{M}^{\prime}\right)$ 作图, 根据广义德拜公式 拟合得到的 Cole-Cole 曲线如图 $5 \mathrm{c}$ 所示 ${ }^{[32]}$. 当温度低于 $4.0 \mathrm{~K}$ 后等温磁化率的 $\chi^{\prime \prime}{ }_{M}$ 峰较宽且 Cole-Cole 曲线越发 偏离半圆形，弛豫时间的分布系数 $\alpha$ 值也随之增大，表 明 $\mathrm{TbNi}_{5} \mathrm{~F}_{2}$ 可能具有较宽的弛豫时间分布以及多种共存 的磁弛豫路径. 结合分子的晶体结构只有一个 $\mathrm{Tb}(\mathrm{III})$ 离 子和 $\left\{\mathrm{Ni}_{5}\right\}$ 环，考虑到低温时反铁磁 $\left\{\mathrm{Ni}_{5}\right\}$ 环对磁化率的 贡献很小, 推测弛豫过程主要来自中心的 $\mathrm{Tb}(\mathrm{III})$ 的单离 子各向异性以及分子内可能存在的 $3 \mathrm{~d}-4 \mathrm{f}$ 磁交换作用. 此外, 分子间最近的 $\mathrm{Tb} \cdots \mathrm{Tb}$ 距离大于 $1.2560 \mathrm{~nm}$ $\left(\mathrm{TbNi}_{5} \mathrm{~F}_{2}\right.$ : $1.4045 \mathrm{~nm} ; \mathrm{TbNi}_{5} \mathrm{Cl}_{2}: 1.2560 \mathrm{~nm} ; \mathrm{TbNi}_{5} \mathrm{Br}_{2}$ : $1.4110 \mathrm{~nm}$ ), 因此分子间 $\mathrm{Tb}(\mathrm{III})$ 离子之间的偶极-偶极相 互作用不大.

为了深入探究 $\mathrm{TbNi}_{5} \mathrm{~F}_{2}$ 的磁弛豫行为, 根据广义德 拜模型从等温磁化率数据提取出与温度有关的自旋一晶 格弛豫时间 $\tau$, 进一步分析 $\tau$ 与 $T$ 的变化关系. 它们的关 系可以表示为:

$$
\tau^{-1}=\mathrm{A} H^{4} T+B_{1} /\left(1+B_{2} H^{2}\right)+C T^{n}+\tau_{0}^{-1} \exp \left(-U_{\text {eff }} / k_{\mathrm{B}} T\right)
$$



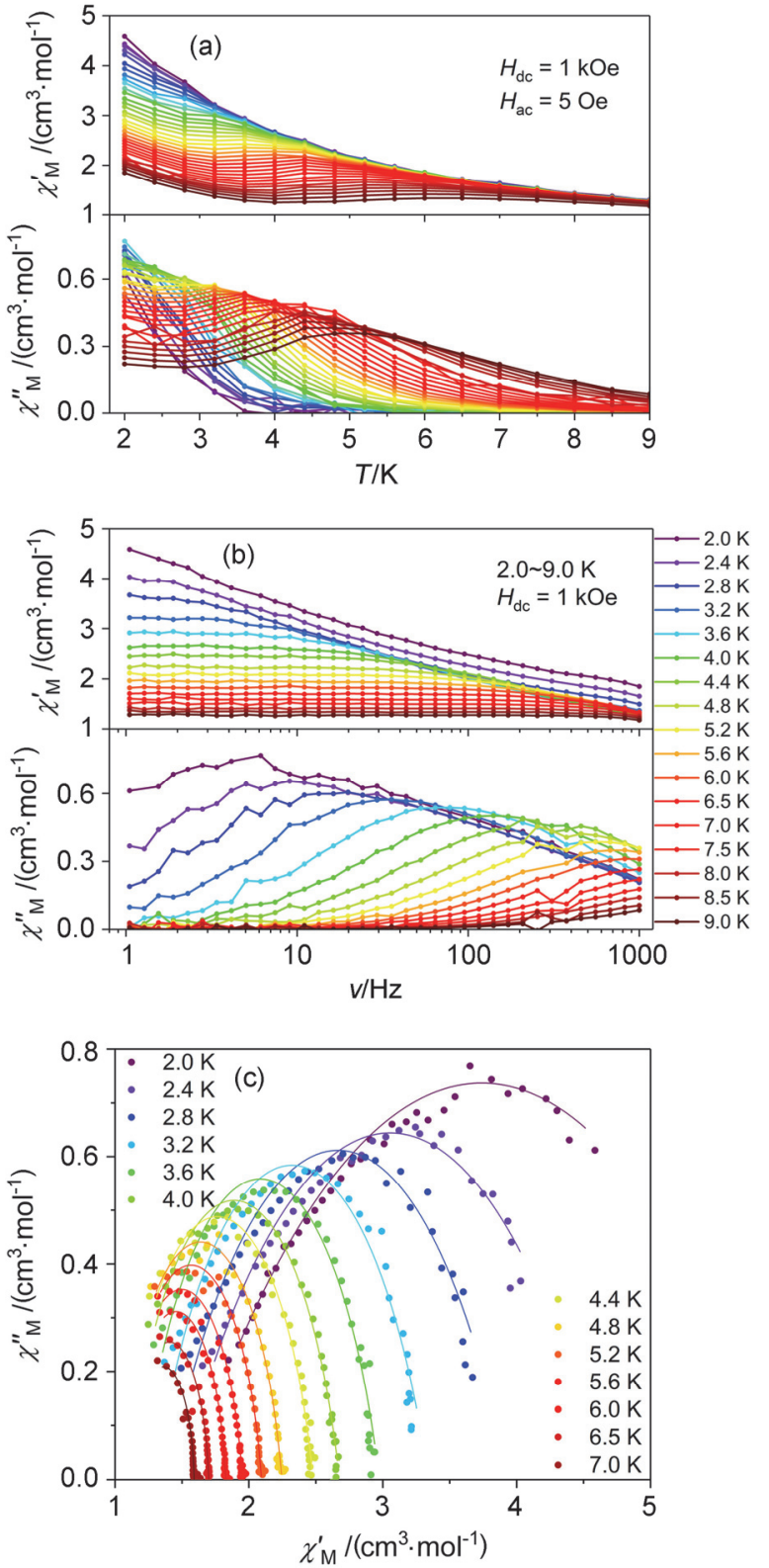

图 5 在 $1 \mathrm{kOe}$ 直流场下 $\mathrm{TbNi}_{5} \mathrm{~F}_{2}$ 的变温 $(\mathrm{a})$ 和变频(b)交流摩尔磁化率 以及 Cole-Cole 曲线图(c)

Figure 5 Temperature-dependent (a) and frequency-dependent (b) AC molar magnetic susceptibilities under $1 \mathrm{kOe}$ DC field and Cole-Cole plots at $2 \sim 7 \mathrm{~K}(\mathrm{c})$ of $\mathrm{TbNi}_{5} \mathrm{~F}_{2}$. Solid lines were fitted using a generalized Debye model

等式右边表示四项不同的弛豫过程，分别为直接(direct) 过程、磁量子隧穿过程、拉曼(Raman)过程和奥巴赫 (Orbach)过程, 其中 $A 、 B_{1} 、 B_{2}$ 和 $C$ 均为化合物的内禀 系数, $H$ 为磁场强度, $k_{\mathrm{B}}$ 为玻尔兹曼常数.

从 $\tau$ 对 $T$ 的双对数坐标图(图 6 左)看出, $2 \sim 6 \mathrm{~K}$ 温区 内 $\tau$ 与 $T$ 的变化关系比较符合 $\tau \propto T^{n}$, 即按拉曼过程拟 合得到 $n=4.6(2)$. 理想情况下, 对非克喇末离子来说, $n=7$; 对克喇末离子来说, $n=9$, 但考虑到实际例子的 声子谱偏离德拜模型, 因此 $n$ 值在 $1 \sim 6$ 范围内都是合理
的 ${ }^{[33]}$. 另外，利用阿伦尼乌斯公式(Arrhenius law)对 $2.8 \sim 6.0 \mathrm{~K}$ 的数据进行拟合(图 6 右), 得到单分子磁体 $\mathrm{TbNi}_{5} \mathrm{~F}_{2}$ 的 “表观能垒” 为 19.0(4) $\mathrm{K}$, 指前因子 $\tau_{0}=$ $8.8(9) \times 10^{-6} \mathrm{~s}$. 值得注意的是, 对包含不同弛豫过程的 全程拟合可能导致过度参数化, 拟合结果难以让人信 服，常常需要结合第一性原理的计算结果. 因此，我们 这里没有对该复杂体系的实际弛豫过程做进一步的归 属和深入研究.
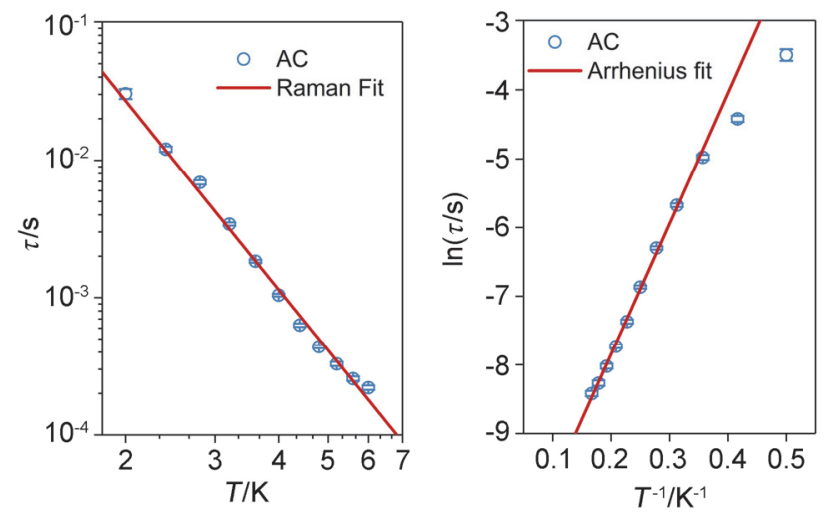

图 $6 \mathrm{TbNi}_{5} \mathrm{~F}_{2}$ 的弛豫时间 $\tau$ 与温度 $T$ (左图)和 $\ln \left(\tau / \mathrm{s}\right.$ ) $-T^{-1}$ (右图)曲线图 (红线分别对应于幂次公式和阿伦尼乌斯公式拟合)

Figure 6 Plot of the relaxation time $\tau$ vs. $T$ (left) and $\ln (\tau / \mathrm{s})$ vs. $T^{-1}$ (right) for $\mathrm{TbNi}_{5} \mathrm{~F}_{2}$ (the red solid lines are the best fits using the power law and the Arrhenius law, respectively)

从 $\mathrm{TbNi}_{5} \mathrm{Cl}_{2}$ 到 $\mathrm{TbNi}_{5} \mathrm{~F}_{2}$, 轴向配位的卤素离子从 $\mathrm{Cl}^{-}$到 $\mathrm{F}^{-}$的改变缩短了 $\mathrm{Tb}-\mathrm{X}$ 键长, 最终影响了整个分 子的慢磁弛豫行为, 即在同样的外场下动态磁行为从较 快的磁弛豫转变为较慢的单分子磁体. 反向考虑, 如果 将轴向配位的卤素离子换成半径更大的 $\mathrm{Br}^{-}$离子, 结果 又会如何？按照这个思路, 我们得到了轴向为 $\mathrm{Br}^{-}$离子 配位的 $\mathrm{TbNi}_{5} \mathrm{Br}_{2}$. 它的赤道面 $\mathrm{Tb}-\mathrm{O}$ 平均键长为 $0.2442(6) \mathrm{nm}$, 与 $\mathrm{TbNi}_{5} \mathrm{~F}_{2}(0.2462(3) \mathrm{nm})$ 和 $\mathrm{TbNi}_{5} \mathrm{Cl}_{2}$ $(0.2436(6) \mathrm{nm})$ 的相差不大. 但轴向 $\mathrm{Tb}-\mathrm{Br}$ 平均键长增 加到约 $0.2825(2) \mathrm{nm}$, 比 $\mathrm{Tb}-\mathrm{Cl}$ 键长更长, 更是远超 $\mathrm{Tb}-\mathrm{F}$ 键长(图 7). 因此, 虽然 $\mathrm{TbNi}_{5} \mathrm{Br}_{2}$ 同样具有五角双 锥构型，但配体的电荷更多地分布于 $\mathrm{Tb}(\mathrm{III})$ 的赤道平 面，进一步削弱了 $\mathrm{Tb}(\mathrm{III})$ 的磁各向异性，由此推测 $\mathrm{TbNi}_{5} \mathrm{Br}_{2}$ 的磁弛豫速率比 $\mathrm{TbNi}_{5} \mathrm{Cl}_{2}$ 还要快. (a)

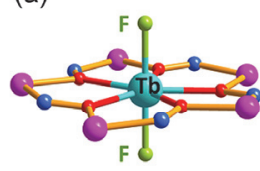

Tb- $\mathrm{F}_{a v}: 0.2152(3) \mathrm{nm}$ $\angle \mathrm{F}-\mathrm{Tb}-\mathrm{F}: 178.91(12)^{\circ}$

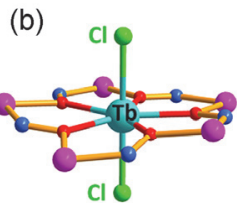

$\mathrm{Tb}-\mathrm{Cl}_{\mathrm{ar}}: 0.2676(1) \mathrm{nm}$ $\angle \mathrm{Cl}-\mathrm{Tb}-\mathrm{Cl}: 176.66(3)^{\circ}$

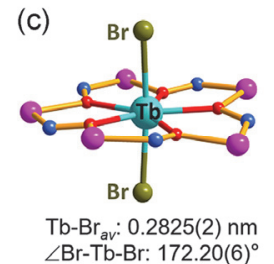

图 7 中心 $\mathrm{Tb}(\mathrm{III})$ 离子的配位环境

Figure 7 Coordination environment of the $\mathrm{Tb}$ (III) ions (a) $\mathrm{TbNi}_{5} \mathrm{~F}_{2}$; (b) $\mathrm{TbNi}_{5} \mathrm{Cl}_{2}$; (c) $\mathrm{TbNi}_{5} \mathrm{Br}_{2}$ 
正如我们所料, 就算外加 $1 \mathrm{kOe}$ 的直流磁场, $\mathrm{TbNi}_{5} \mathrm{Br}_{2}$ 的交流磁化率曲线都没表现出频率依赖性(图 $8)$. 而且在 $2 \sim 8 \mathrm{~K}$ 温区内 $\chi^{\prime \prime}{ }_{\mathrm{M}}$ 值基本没有响应. 因此, 对 $\mathrm{TbNi}_{5} \mathrm{Br}_{2}$ 而言无论是否外加磁场, 都可认为它没有表 现出慢磁弛豫行为.

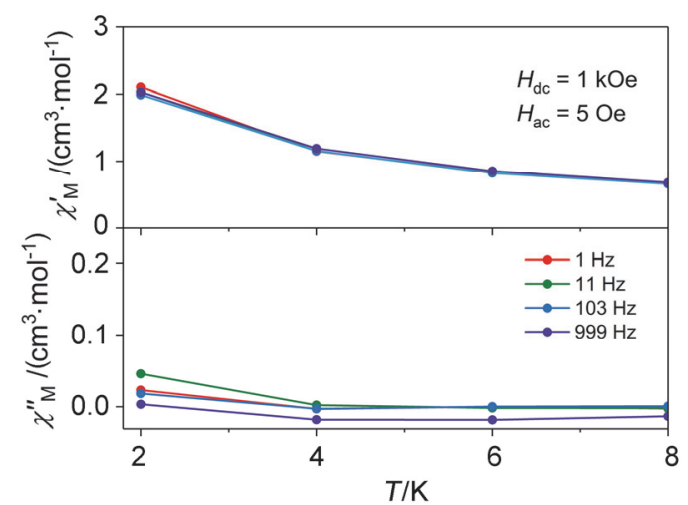

图 8 在 $1 \mathrm{kOe}$ 直流外场下 $\mathrm{TbNi}_{5} \mathrm{Br}_{2}$ 的变温交流摩尔磁化率曲线

Figure 8 Temperature-dependent $\mathrm{AC}$ molar magnetic susceptibilities of $\mathrm{TbNi}_{5} \mathrm{Br}_{2}$ under $1 \mathrm{kOe}$ DC field

其实, 对 $D_{5 h}$ 对称性来说, 轴向 $\mathrm{X}-\mathrm{Tb}-\mathrm{X}$ 键角的 贡献也不能忽视. 与 $\mathrm{Cl}-\mathrm{Tb}-\mathrm{Cl}$ 键角 $176.66(3)^{\circ}$ 相比, $\mathrm{F}-\mathrm{Tb}-\mathrm{F}$ 键角 $178.91(12)^{\circ}$ 更接近线性 $180^{\circ}$, 而 $\mathrm{Br}-$ $\mathrm{Tb}-\mathrm{Br}$ 的键角 $172.22(6)^{\circ}$ 偏离线性最大(图 7). 换句话 说, $\mathrm{TbNi}_{5} \mathrm{~F}_{2}$ 中稀土离子的配位几何更接近理想的五角 双雉构型，所以更适合扁圆形的 Tb(III)离子.

\section{3 结论}

基于金属冠醚[15-MC $\left.\mathrm{Mi}_{\mathrm{Ni}(\mathrm{II})}-5\right]$, 报道了三例混合过渡 金属一稀土配合物 $\left\{\mathrm{TbNi}_{5} \mathrm{X}_{2}\right\}(\mathrm{X}=\mathrm{F}, \mathrm{Cl}, \mathrm{Br})$. 中心 $\mathrm{Tb}(\mathrm{III})$ 离子均采取赝 $D_{5 h}$ 对称性的五角双雉配位几何构型, 两 个卤素离子 $\mathrm{X}^{-}$占据上下轴向位置, 形成罕见的线形 $\{\mathrm{X}-\mathrm{Tb}-\mathrm{X}\}$ 基元. 动态磁学分析表明 $\mathrm{TbNi}_{5} \mathrm{Cl}_{2}$ 具有慢 磁弛豫行为. 以它为参考, 当轴向配位半径更小的 $\mathrm{F}^{-}$离 子时, $\mathrm{TbNi}_{5} \mathrm{~F}_{2}$ 表现出单分子磁体行为; 反之, 轴向配位 半径更大的 $\mathrm{Br}^{-}$离子时, 慢磁弛豫行为消失. 原因是 $\mathrm{Tb}(\mathrm{III})$ 离子的 $4 \mathrm{f}$ 电子云呈扁圆形分布, 轴向电荷分布越 靠近 $\mathrm{Tb}(\mathrm{III})$, 越有利于提高稀土离子的磁各向异性. 因 此, 对具有 $D_{5 h}$ 对称性的 $\left\{\mathrm{TbNi}_{5} \mathrm{X}_{2}\right\}$ 来说, 可以通过引入 电负性不同的卤素离子调节稀土离子的轴向电荷分布, 从而实现调控分子动态磁行为的目的. 我们推测, 含其 它稀土离子(如 Dy(III)、Ho(III)等)的线形 $\{\mathrm{F}-\mathrm{Ln}-\mathrm{F}\}$ 基 元体系将会具有新奇的磁弛豫行为, 进一步的研究正在 进行中.

\section{4 实验部分}

\section{1 仪器和试剂}

本实验所用药品和试剂均为市售分析纯级别, 如无 特殊说明均未经预处理直接使用. 喹啉羟肜酸配体的合
成参考文献 [29]报道的方法. 傅里叶变换红外光谱 (FT-IR)采用溴化钾压片的方法在 Bruker EQUINOX 55 型红外光谱仪测量, 测试范围为 $4000 \sim 400 \mathrm{~cm}^{-1}$. 化合 物 $\mathrm{C}, \mathrm{H}, \mathrm{N}$ 的元素分析(EA)数据采用 Elemental Vario-EL Cube 元素分析仪测量. 磁性测试样品取自实验合成的 晶体样品, 采用 Quantum Design MPMS3 磁学测量系统 测试. 直流磁化率的测试温度为 $2 \sim 300 \mathrm{~K}$, 磁场强度为 0 7 $\mathrm{T}$; 交流磁化率测试的振荡磁场强度为 $5 \mathrm{Oe}$, 直流 磁场强度为 $1 \mathrm{kOe}$. 测试过程中使用凡士林包裹预处理 后的样品, 防止晶体在磁场下发生转动, 磁学数据均已 进行抗磁校正.

\section{2 配合物的合成}

4.2.1 $\left[\mathrm{TbNi}_{5} \mathrm{~F}_{2} \text { (quinha) }\right)_{5}$ (3-chloropyridine $\left.\left.)_{10}\right)\right]\left(\mathrm{ClO}_{4}\right)_{0.5^{-}}$ $\mathrm{Cl}_{0.5} \cdot 10 \mathrm{H}_{2} \mathrm{O}\left(\mathrm{TbNi}_{5} \mathrm{~F}_{2}\right)$ 的合成

将六水合氯化铽 $(0.02 \mathrm{mmol})$ 、六水合高氯酸镍 $(0.10$ $\mathrm{mmol})$ 及喹啉羟肜酸 $(0.10 \mathrm{mmol})$ 加入到 $25 \mathrm{~mL}$ 特氟龙内 祄的不锈钢反应釜中, 再加入 $8 \mathrm{~mL}$ 无水甲醇和 $1 \mathrm{~mL} 3-$ 氯吡啶. 待溶液搅拌溶解后, 加入氟化铵 $(0.10 \mathrm{mmol})$ 和 三乙胺 $(0.20 \mathrm{mmol})$, 黄绿色混合溶液马上变成深红色. 将反应釜置于 $75{ }^{\circ} \mathrm{C}$ 恒温烘箱中 $48 \mathrm{~h}$, 冷却至室温后得 $12 \mathrm{mg}$ 红色块状晶体(21.4\%). IR (KBr) v: 3358, 3066, $1673,1602,1583,1541,1510,1464,1417,1367,1338$, 1304, 1213, 1182, $1093\left(\mathrm{ClO}_{4}^{-}\right), 1049,974,941,847,802$, 764, 737, 694, 636, 617, 545, 519, $496 \mathrm{~cm}^{-1}$. Anal. calcd for $\mathrm{C}_{100} \mathrm{H}_{90} \mathrm{~N}_{20} \mathrm{~F}_{2} \mathrm{Cl}_{11} \mathrm{Ni}_{5} \mathrm{TbO}_{22}$ : C 42.83, H 3.23, N 9.99; found: C 42.57, H 3.12, N 10.25.

\subsection{2 $\left.\left[\mathrm{TbNi}_{5} \mathrm{Cl}_{2} \text { (quinha }\right)_{5}(\text { pyridine })_{10}\right]\left(\mathrm{BPh}_{4}\right) \cdot 2 \mathrm{MeOH} \bullet$} pyridine $\cdot 5 \mathrm{H}_{2} \mathrm{O}\left(\mathrm{TbNi}_{5} \mathrm{Cl}_{2}\right)$ 的合成

将六水合氯化铽 $(0.02 \mathrm{mmol})$ 、六水合高氯酸镍 $(0.10$ $\mathrm{mmol})$ 及喹啉羟肟酸 $(0.10 \mathrm{mmol})$ 加入到 $25 \mathrm{~mL}$ 特氟龙内 祄的不锈钢反应釜中, 再加入 $8 \mathrm{~mL}$ 无水甲醇和 $1 \mathrm{~mL}$ 吡 啶. 待溶液混合均匀后, 依次加入四苯硼钠 $(0.02 \mathrm{mmol})$ 和三乙胺 $(0.20 \mathrm{mmol})$. 将反应釜置于 $75{ }^{\circ} \mathrm{C}$ 恒温烘箱中 $48 \mathrm{~h}$, 冷却至室温后得 $18 \mathrm{mg}$ 红色块状晶体(32.2\%). IR (KBr) v: 3388, 3064, 1693, 1587, 1545, 1510, 1485, 1443, 1369, 1338, 1302, 1215, 1182, 1149, 1117, 1045, 974, 939, $847,758,700,640,617,546,521,494 \mathrm{~cm}^{-1}$. Anal. calcd for $\mathrm{C}_{131} \mathrm{H}_{123} \mathrm{BN}_{21} \mathrm{Cl}_{2} \mathrm{Ni}_{5} \mathrm{TbO}_{17}$ : C 56.24, $\mathrm{H}$ 4.43, $\mathrm{N} 10.51$; found: C 55.86, H 4.14, N 10.62.

4.2.3 $\left[\mathrm{TbNi}_{5} \mathrm{Br}_{2} \text { (quinha) }\right)_{5}$ (3-bromopyridine) $\left.)_{10}\right] \mathrm{Br} \bullet 2 \mathrm{MeOH} \bullet$ $\mathrm{H}_{2} \mathrm{O}\left(\mathrm{TbNi}_{5} \mathrm{Br}_{2}\right)$ 的合成

将溴化铽 $(0.04 \mathrm{mmol}) 、$ 溴化镍 $(0.20 \mathrm{mmol})$ 及喹啉羟 肜酸 $(0.20 \mathrm{mmol})$ 加入到 $25 \mathrm{~mL}$ 特氟龙内衬的不锈钢反

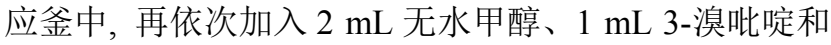
三乙胺 $(0.40 \mathrm{mmol})$. 将反应釜置于 $75{ }^{\circ} \mathrm{C}$ 恒温烘箱中 48 $h$, 冷却至室温后得 $20 \mathrm{mg}$ 红色条状晶体(15.2\%). IR (KBr) v: 3373, 3062, 1603, 1585, 1543, 1510, 1464, 1416, 1367, 1340, 1313, 1260, 1213, 1182, 1117, 1092, 1045, 
1024, 974, 939, 847, 795, 762, 694, 633, 615, 546, 519, $494 \mathrm{~cm}^{-1}$. Anal. calcd for $\mathrm{C}_{102} \mathrm{H}_{80} \mathrm{~N}_{20} \mathrm{Ni}_{5} \mathrm{Br}_{13} \mathrm{TbO}_{13}$ : C 37.29, H 2.45, N 8.53; found: C 37.63, H 2.49, N 8.47.

\section{3 单晶结构测定}

配合物的单晶结构采用 Bruker D8 QUEST X 射线 单晶衍射仪测试(Mo K $\alpha$ radiation, $\lambda=0.071073 \mathrm{~nm}$ ), 在 $120 \mathrm{~K}$ 下收集衍射数据, 运用 SAINT 程序还原数据和 SADABS 程序进行经验吸收校正 ${ }^{[34,35]}$. 晶体结构解析和 精修采用 SHELXT 和 Olex2 程序包处理 ${ }^{[36 ~ 38]}$. 三个化合 物的 CCDC 号为 $1945892 \sim 1945894$.

由于晶格中部分辅助配体(3-氯吡啶和 3-溴吡啶)的 卤素原子以及未配位水分子严重无序, 所以有些溶剂分 子没有加氢. 剩余残余峰为无序的溶剂分子, 经过 SQUEEZE 程序处理 ${ }^{[39]}$, 再参考 SHELX 晶体解析软件 给出的非氢原子平均体积, 根据元素分析结果推测出残 余峰对应的溶剂分子数目, 进一步确定化合物精确的分 子式.

\section{References}

[1] Sessoli, R.; Gatteschi, D.; Caneschi, A.; Novak, M. A. Nature 1993, $365,141$.

[2] Gatteschi, D.; Sessoli, R.; Villain, J. Molecular Nanomagnets, Oxford University Press, Oxford, 2006.

[3] Meng, Y.-S.; Jiang, S.-D.; Wang, B.-W.; Gao, S. Acc. Chem. Res. 2016, 49, 2381.

[4] Ungur, L.; Lin, S.-Y.; Tang, J.; Chibotaru, L. F. Chem. Soc. Rev. 2014, 43, 6894 .

[5] McClain, K. R.; Gould, C. A.; Chakarawet, K.; Teat, S. J.; Groshens, T. J.; Long, J. R.; Harvey, B. G. Chem. Sci. 2018, 9, 8492.

[6] Gould, C. A.; McClain, K. R.; Yu, J. M.; Groshens, T. J.; Furche, F.; Harvey, B. G.; Long, J. R. J. Am. Chem. Soc. 2019, 141, 12967.

[7] Goodwin, C. A.; Ortu, F.; Reta, D.; Chilton, N. F.; Mills, D. P. Nature 2017, 548, 439.

[8] Guo, F. S.; Day, B. M.; Chen, Y. C.; Tong, M. L.; Mansikkamaki, A.; Layfield, R. A. Angew. Chem., Int. Ed. 2017, 56, 11445.

[9] Guo, F.-S.; Day, B. M.; Chen, Y.-C.; Tong, M.-L.; Mansikkamaki, A.; Layfield, R. A. Science 2018, 362, 1400.

[10] Guo, P.-H.; Liao, X.-F.; Leng, J.-D.; Tong, M.-L. Acta Chim. Sinica 2013, 71, 173. (郭鹏虎, 廖小芬, 冷际东, 童明良, 化学学报, 2013, 71, 173.)

[11] Tian, H.-Q.; Zheng, L.-M. Acta Chim. Sinica 2020, 78, 34. (田海权, 郑丽敏, 化学学报, 2020, 78, 34.)

[12] Velkos, G.; Krylov, D. S.; Kirkpatrick, K.; Spree, L.; Dubrovin, V.; Bgchner, B.; Avdoshenko, S. M.; Bezmelnitsyn, V.; Davis, S.; Faust, P.; Duchamp, J.; Dorn, H. C.; Popov, A. A. Angew. Chem., Int. Ed. 2019, $58,5891$.
[13] Li, H.; Meng, X.; Wang, M.; Wang, Y.-X.; Shi, W.; Cheng, P. Chin. J. Chem. 2019, 37, 373.

[14] Gou, X.-S.; Wang, M.-M.; Meng, Q.-Q.; Cheng, P. Chin. J. Inorg. Chem. 2019，35, 2013. (芶晓霜，王梦梦，孟茜茜，程鹏，无机化 学学报, 2019, 35, 2013.)

[15] Yan, H.; Li, Q.-W.; Liu, J.-L.; Jia, J.-H.; Tong, M.-L. J. Chin. Soc. Rare Earths 2016, 34, 726. (严华，陈龑骢，李泉文，刘俊良，贾建 华, 童明良, 中国稀土学报, 2016, 34, 726.)

[16] Liu, K.; Zhang, X.; Meng, X.; Shi, W.; Cheng, P.; Powell, A. K. Chem. Soc. Rev. 2016, 45, 2423.

[17] (a) Sievers, J. Z. Phys. B: Condens. Matter 1982, 45, 289. (b) Rinehart, J. D.; Long, J. R. Chem. Sci. 2011, 2, 2078.

[18] Liu, J.-L.; Chen, Y.-C.; Zheng, Y.-Z.; Lin, W.-Q.; Ungur, L.; Wernsdorfer, W.; Chibotaru, L. F.; Tong, M.-L. Chem. Sci. 2013, 4, 3310 .

[19] Liu, J.-L.; Chen, Y.-C.; Tong, M.-L. Chem. Soc. Rev. 2018, 47, 2431.

[20] Liu, J.-L.; Wu, J.-Y.; Chen, Y.-C.; Mereacre, V.; Powell, A. K.; Ungur, L.; Chibotaru, L. F.; Chen, X.-M.; Tong, M.-L. Angew. Chem., Int. Ed. 2014, 53, 12966.

[21] Huang, G.-Z.; Ruan, Z.-Y.; Zheng, J.-Y.; Chen, Y.-C.; Wu, S.-G.; Liu, J.-L.; Tong, M.-L. Sci. China, Chem. 2020, 63, DOI: 10.1007/ s11426-020-9746-x.

[22] Huang, G.- Z.; Ruan, Z. Y.; Zheng, J. Y.; Wu, J. Y.; Chen, Y. C.; Li, Q. W.; Akhtar, M. N.; Liu, J. L.; Tong, M. L. Sci. China, Chem. 2018, 61, 1399.

[23] Liu, J.; Chen, Y. C.; Liu, J. L.; Vieru, V.; Ungur, L.; Jia, J. H.; Chibotaru, L. F.; Lan, Y. H.; Wernsdorfer, W.; Gao, S.; Chen, X. M.; Tong, M. L. J. Am. Chem. Soc. 2016, 138, 5441.

[24] Chen, Y. C.; Liu, J. L.; Ungur, L.; Liu, J.; Li, Q. W.; Wang, L. F.; Ni, Z. P.; Chibotaru, L. F.; Chen, X. M.; Tong, M. L. J. Am. Chem. Soc. 2016, 138, 2829.

[25] Chen, Y.-C.; Liu, J.-L.; Wernsdorfer, W.; Liu, D.; Chibotaru, L. F.; Chen, X. M.; Tong, M. L. Angew. Chem., Int. Ed. 2017, 56, 4996.

[26] Mezei, G.; Zaleski, C. M.; Pecoraro, V. L. Chem. Rev. 2007, 107, 4933.

[27] Happ, P.; Plenk, C.; Rentschler, E. Coord. Chem. Rev. 2015, 289, 238.

[28] Li, Q.-W.; Liu, J.-L.; Jia, J.-H.; Chen, Y.-C.; Liu, J.; Wang, L.-F.; Tong, M.-L. Chem. Commun. 2015, 51, 10291.

[29] Li, Q.-W.; Wan, R.-C.; Chen, Y.-C.; Liu, J.-L.; Wang, L.-F.; Jia, J.-H.; Chilton, N. F.; Tong, M.-L. Chem. Commun. 2016, 52, 13365.

[30] Carlin, R. L. Translated by Wan, C. D. Magnetochemistry, Nanjing University Press, Nanjing, 1990. (Carlin, R. L., 磁化学, 万纯娣译, 南京大学出版社, 南京, 1990.)

[31] Wang, J.; Ruan, Z.-Y.; Li, Q.-W.; Chen, Y.-C.; Huang, G.-Z.; Liu, J.-L.; Reta, D.; Chilton, N. F.; Wang, Z.-X.; Tong, M.-L. Dalton Trans. 2019, 48, 1686.

[32] Cole, K. S.; Cole, R. H. J. Chem. Phys. 1941, 9, 341.

[33] Liu, J.-L.; Yuan, K.; Leng, J.-D.; Ungur, L.; Wernsdorfer, W.; Guo, F.-S.; Chibotaru, L. F.; Tong, M.-L. Inorg. Chem. 2012, 51, 8538.

[34] Bruker, SAINT, Version V8. 37A, Madison, 2015.

[35] Bruker, $S A D A B S$, Version 2014/5, Madison, 2014.

[36] Bruker, SHELXTL, Software Version 6. 12, Madison, 2001.

[37] Sheldrick, G. M. Acta Cryst. 2008, A64, 112.

[38] Dolomanov, O. V.; Bourhis, L. J.; Gildea, R. J.; Howard, J. A. K.; Puschmann, H. J. Appl. Crystallogr. 2009, 42, 339.

[39] Spek, A. L. Acta Cryst. 2015, C71, 9.

(Zhao, C.) 